\title{
Gingival Transcriptomics of Follicular T Cell Footprints in Progressing Periodontitis
}

\author{
Jeffrey Ebersole ${ }^{1}$, Sreenatha Kirakodu², Luis Orraca ${ }^{3}$, Janis Gonzalez-Martinez ${ }^{4}$, and \\ Octavio Gonzalez ${ }^{2}$ \\ ${ }^{1}$ University of Nevada Las Vegas \\ ${ }^{2}$ University of Kentucky \\ ${ }^{3}$ University of Puerto Rico Medical Sciences Campus \\ ${ }^{4}$ University of Puerto Rico
}

September 10, 2020

\begin{abstract}
Follicular helper $\mathrm{T}$ cells ( $\mathrm{Tfh}$ ) cells are generally considered critical in secondary lymphoid tissues; however, they are also identified in the circulation and in tertiary lymphoid structures in chronic inflammation. Gingival tissues with periodontitis reflect chronic inflammation so genomic footprints of Tfh cells should occur in these tissues and may differ related to aging effects. Methods: Macaca mulatta monkeys were used in a model of ligature-induced periodontitis [adult group (12-23 years of age); young group (3-7 years)]. Gingival tissue and subgingival microbiome samples were obtained at matched healthy sites, sites during ligature-induced disease, and in samples after clinical resolution. Microarray analysis examined Tfh genes ( $\mathrm{n}=40$ ) and the microbiome samples were examined using 16S MiSeq. Results: An apparent increase in the major transcription factor of Tfh cells, BCL6, was found with disease in both adult and young animals, while the master transcription markers of other T cell subsets were either decreased or showed minimal change. A number of the Tfh related genes, including surface receptors, secreted products and transcription factors were also significantly increased during disease. Unique microbial complexes showed patterns of interactions with Tfh genes that differed in health and disease. Conclusions: An increase in Tfh cell responsiveness occurred later in the progression of periodontitis, affected by age and strongly related to specific microbial complexes. The capacity of gingival Tfh cells to contribute to localized B cell activation and active antibody responses, including affinity maturation may be critical for controlling periodontal lesions and contributing to limiting and/or resolving the lesions.
\end{abstract}

\section{Introduction}

Follicular helper $\mathrm{T}$ cells are a subset of $\mathrm{CD} 4^{+}$helper cells that are involved in the regulation and development of antigen-specific B cell immunity. The fundamental function of $\mathrm{T}$ helper cells is to provide help to $\mathrm{B}$ cells and to regulate their proliferation and immunoglobulin class switching, with antibody affinity maturation. Historically, Tfh cells were considered to be generally localized to the B cell follicle of secondary lymphoid tissues, including tonsils, to interact with germinal center (GC) B cells [1-3]. Functional studies have demonstrated that Tfh cells provide signals that are essential for survival and proliferation of these GC B cells. Thus, they play a critical role in development of B cell protective immunity against pathogens via immunoglobulin switching and recombination to promote increased antibody affinity [2].

Tfh differentiation is a multi-stage process and their functions depend upon the master regulatory transcription factor B-cell lymphoma 6 (Bcl6) [4-6]. Bcl-6 also suppresses the expression of factors that promote the differentiation of other $\mathrm{CD} 4^{+} \mathrm{T}$ helper cell subsets[7]. Canonical secreted $\mathrm{Tfh}$ molecules include CXCL13, IL-21 and IL-4 that are all important for B cell help. IL-21 also regulates CD4 ${ }^{+} \mathrm{T}$ cell subsets, including the efficient development of Tfh, as well as B cell differentiation and activation. Tfh cells also 
display crucial cell surface markers, including CXCR5, PD1, ICOS, and CD40LG. The early differentiation of Tfh is driven by IL-6, ICOS, and the T cell receptor (TCR). The majority of differentiated Tfh cells

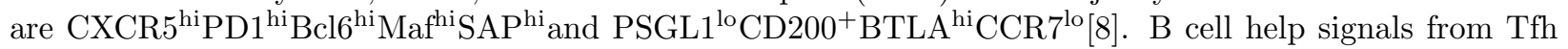
include both cytokines and cell surface receptors [9]. Coupled with CD40LG, both PD1 and ICOS bind to ligands on the $\mathrm{B}$ cell surface to regulate the functions of both the B cells and Tfh cells, with direct co-stimulation of B cells via CD40LG and production of IL-21 driving B cell proliferation. The CXCR5 receptor enhances chemoattraction and migration of the Tfh cells into GC B cell zones[10-12].

Of particular interest to this study is that both circulating and tissue resident Tfh cells can be detected that produce cytokines to modulate antibody responses. Moreover, tissues including the periodontium, that contain antigens in a milieu of chronic immune responses are often infiltrated by T cells, macrophages, B cells and plasmacytes [13]. These cellular elements can organize themselves anatomically and functionally in a manner similar to secondary lymphoid organs [13-15]. As the gingival tissues with periodontitis reflect this type of microenvironment we hypothesized that with development and progression of periodontitis, genomic footprints of Tfh cells would occur in the gingival tissues and may differ related to aging effects. It was also predicted that certain bacterial components of the complex oral microbiome would have the capacity to aid in triggering or dampening the various molecules required for functional Tfh cells in the gingival tissues.

\section{Methods}

\section{Animals and Diet}

Rhesus monkeys (Macaca mulatta) ( $\mathrm{n}=36 ; 17$ male, 19 female) housed at the Caribbean Primate Research Center at Sabana Seca, Puerto Rico were examined for periodontal health [16-18]. Adult animals (age: 12-23 years; $n=18$ ) and young animals (age: $3-7$ years; $n=18$ ) were included in the study. The nonhuman primates were fed a $20 \%$ protein, $5 \%$ fat, and $10 \%$ fiber commercial monkey diet (diet 8773 , Teklad NIB primate diet modified: Harlan Teklad, Madison, WI). The diet was supplemented with fruits and vegetables, and water was provided ad libitum in an enclosed corral setting.

As we have reported previously the protocol was approved by the Institutional Animal Care and Use Committee (IACUC) of the University of Puerto Rico and a ligature disease model was utilized [19]. The clinical examination included probing pocket depth (PPD) and bleeding on probing (BOP; 0-5 scale) [20]. Periodontal health was defined by mean Pocket Depth (PD) [?] $3.0 \mathrm{~mm}$ and mean Bleeding on Probing (BOP) [?] 1 (0-5 scale) in a full mouth examination excluding $3^{\text {rd }}$ molars and canines [19]. Ligature-induced periodontal disease was initiated as we have previously reported [19] and gingival and subgingival plaque samples taken at $0.5,1$, and 3 months (Initiation/Progression), and 2 months after removal of ligatures and local factors (Resolution). Determination of periodontal disease at the sampled site was documented by assessment of the presence of $\mathrm{BOP}$ and probing pocket depth of $>4 \mathrm{~mm}$, as we have described previously [17].

\section{Microbiome analysis}

Subgingival bacterial samples were obtained from the 36 animals by a curette and analyzed using a MiSeq instrument $[21,22]$ for the total composition of the microbiome from each sample [23, 24]. Sequences were clustered into phylotypes based on their sequence similarity and these binned phylotypes were assigned to their respective taxonomic classification using the Human Oral Microbiome Database (HOMD V13) (http://www.homd.org/index.php?name=seqDownload \&file\&type=R) as we have described previously [25]. Raw data were deposited at the NIH NCBI (BioProject ID PRJNA516659). Statistical differences of bacterial OTUs were determined with a $t$-test $(\mathrm{p}<0.05)$. Correlations of OTUs within the oral microbiome were determined using a Pearson correlation coefficient analysis $(\mathrm{p}<0.05)$. Correlations between the microbiome components and the gingival gene expression were determined only for matching samples derived from the same tooth in each of the animals. Matching samples with sufficient microbiome signals were compared for 58 samples in adults and 25 samples from the young group obtained at health and throughout the ligature model. As we have reported previously [25], of 396 OTUs identified in the nonhuman primate oral samples the targeted OTU selection for this study was 58 for the adult samples that covered $88 \%$ of reads in all samples. Similarly, 49 OTUs were examined in the young samples that covered $91 \%$ of reads in all samples. 


\section{Gingival tissue sample collection and $m R N A$ analysis}

Gingival tissue samples of healthy and disease sites were surgically collected and total RNA extracted for microarray analysis via hybridization to the GeneChipß Rhesus Gene 1.0 ST Array (Affymetrix, Santa Clara, CA, USA) similar to methods we have described previously [17, 26-29] .

\section{Data Analysis}

Tfh genes $(\mathrm{n}=40)$ (Table 1 ) were targeted in this study. The expression intensities across the samples were estimated using the Robust Multi-array Average (RMA) algorithm with probe-level quintile normalization, as implemented in the Partek Genomics Suite software version 6.6 (Partek, St. Louis, MO). The age groups were initially compared using one way ANOVA. For genes that had significant mean differences, two sample t-tests were used to investigate differences. Statistical significance was considered by a p value [?] 0.05 . The data has been uploaded into the ArrayExpress data base (www.ebi.ac.uk) under accession number: E-MTAB-1977.

\section{Results}

\section{Tfh gene expression profiles with age and periodontitis}

Figure 1 summarizes fold-changes in gene expression for the $40 \mathrm{Tfh}$ genes that were examined in gingival tissues from young (Fig. 1A ) and adult (Fig. 1B ) animal gingival tissues compared to baseline healthy tissue samples. The result demonstrated a number of the Tfh related genes, including surface receptors, secreted products and transcription factors were significantly increased during disease. In the young animals, striking increases were observed with BTLA, CXCL13 and IRF4 expression levels, while IFNG, CD40LG and CD200 were all significantly decreased. There was considerable overlap in altered gene expression levels in the young and adult samples. BTLA, CXCL13 and IRF4 were also increased and CD200 decreased with disease in the adult animals. Also of note was the increase in BCL6 in both groups primarily at the 3 month time point late in disease progression. The overall pattern of Tfh gene changes in both groups presented at 1 and 3 months during disease progression.

Figure 2 provides a summary of gene expression changes targeting the master regulatory transcription genes for each of the subsets of $\mathrm{T}$ helper cells. In both the adult and young gingival tissues samples the primary increase in transcription factor expression was with BCL6 for Tfh cells, particularly in the latter phases of disease progression, with levels returning to baseline in resolution samples. Also of note was the significant decreases in RORA in both age groups, and RORC (ROR $\gamma$ ) in the young animal tissues through disease initiation and progression, and even in resolution samples. Th1 transcription factor, T-bet/TXB21, expression was only increased in the resolution samples.

Gene expression comparison in adult and young samples

Figure 3A-E provide an analysis of the array of gene changes comparing responses in adults and young animals in health (BL), disease (0.5, 1, 3 months), and resolution (5 months). The data was transformed using a z-score to normalize across all adult and young samples and across all data points. Fig. 3A-B summarizes the response profiles to Tfh-associated surface receptors. Generally, in the young samples the expression level was lower than adults, and did not show much change through the disease process and resolution samples. Only CD200 appeared elevated in young animals and decreased throughout disease. In contrast, in adults the majority of these receptors showed increases through disease and even in the resolution samples compared to baseline. Only CD10 and IL6RA demonstrated an increased with disease initiation (0.5 months) that tended to decrease with disease progression and resolution, and CD57 showed limited changes overtime.

Fig. 3C-D presents a similar analysis for cytokine/chemokine expression. In adults, IL10 and IL17F decreased from baseline, and IL21 and CXCL13 each increased with the disease process. In comparison with the young samples IFNG decreased with disease and CXCL13 and IL4 were increased in disease samples. 
The expression of crucial transcription factors and adaptor molecules for Tfh functions is shown in Figure 3E. Both IRF4 and SH2D1A/SAP showed substantial increases as disease progressed in both adults and young samples with dominant expression in the adult samples. BCL6 increased in adult samples at disease initiation, while this crucial transcription factor decreased in the young samples with disease progression. STAT3 demonstrated an increase at disease initiation and ASCL2 at disease resolution, in the young animal samples.

\section{Microbiome and host Tfh gene expression in adult and young animals samples}

Table 2 provides a summary of the microbiome relationships to gingival tissue transcriptome in samples from the two groups of animals. The data presents complexes of bacterial OTUs that were significantly correlated with individual genes related to Tfh phenotype and functions. An initial comparison of the Tfh transcriptome genes and the microbiome across all adult and young samples demonstrated few significant correlations within the potential 2320 comparisons, with only $2 \%$ showing a significant correlation (data not shown). However, the complexes and relationships were substantially different in health, disease and resolution in adult or young animals samples. Additionally, the same complexes were significantly related to multiple Tfh genes with both positive and negative correlations (see examples Tfh-H3A, Tfh-R4A), while during disease the complexes were only positively correlated with gene expression levels. Similarities on the component members of the complexes showed some overlap in adults (see Tfh-H1A/Tfh-D2A; TfhH4A/Tfh-D3A), although these similarities did not appear in the microbiome complexes in the young animals. Moreover, minimal overlap was noted in the complexes between adult and young animals. In adult animals, the complexes were associated with multiple genes in health, disease and resolution; however, in the young samples, generally, only with the disease associated microbiome were multiple gene correlations observed. Finally, IL6RA, CD84 and CD10 were the Tfh related genes showing the most frequent correlations with the microbial complexes. IL6RA and CD84 were predominantly associated with the microbiome in adults, while CD10 related to microbiome complexes in both adult and young samples.

In addition to exploring the relationship between bacterial complexes and profiles of host Tfh genes with related abundance or expression levels, we identified the individual bacterial OTUs that predominated among these correlations (Table 3 ). The results showed that multiple bacteria historically associated with changes in the disease microbiota, including Fretibacterium species, Treponemaspecies, Porphyromonas species, and Prevotella species demonstrated a high frequency of correlations with Tfh genes. Also of note, Haemophilus, Leptotrichia, and G. morbillorumgenerally considered as commensals also demonstrated an elevated prevalence of correlations with the Tfh gene patterns. A number of the bacteria also appeared to primarily correlate with Tfh gene expression in adult samples compared to young samples. Finally, the was a predominance of individual bacterial-host correlations in resolution samples compared to health or disease in the young animals.

\section{Profiles of microbiome and host Tfh gene expression}

Although we were able to identify complexes that were significantly correlated with host Tfh genes, this approach did not provide a full picture of the potential host-bacterial interactions that occur in the adult and young samples. Figure 4A-C provides a heatmap of the overall bacterial-gene correlations, and highlight specific bacterial complexes and associated targeted gene expression profiles. In young healthy samples there existed correlations with multiple bacterial complexes and numerous host genes showing both positive and negative relationships for the same bacterial complex. A similar pattern was noted in the resolution samples suggesting a close interaction of host responses with the oral microbiome in these types of clinical conditions in younger animals. However, during disease, the bacterial components making up the complexes were more limited, and fewer Tfh genes appeared to be responding to these complexes, with both positive and negative correlation patterns. The adult samples (Figure 5A-C ) generally showed fewer organized bacterial complexes related to Tfh gene expression patterns in health, disease and resolution. In healthy adult samples 4 complexes of bacteria showed patterns of correlations with 3 of the complexes demonstrating these interactions with a small repertoire of the Tfh genes. These host-bacterial interactions were more limited in adult disease samples with 3 complexes all generally correlated with an overlapping set of genes. The adult 
resolution samples showed 5 identified complexes. Three of these complexes demonstrated a relationship to an overlapping set of genes. Of interest was that complex 4 exhibited a very broad negative correlation pattern to 20/40 Tfh genes, while complex 5 showed a strong negative correlation to only CD57/B3GAT1.

Figure 6 provides a schematic of a protein interaction network related to the Tfh genes examined that significantly correlated with bacterial complexes. This includes interactions of transcription factors, cell surface receptors, and soluble products produced by Tfh cells. We highlight the genes expression profiles that were altered in adults and young animal samples stratified into health, disease, and resolution samples. These findings showed that cell receptors CD40LG, CD10/MME, IL6RA, CD84, and the composite of CD3D/E/G were particularly enriched for these correlations. Additionally, of the cytokines/chemokines IL12 and IL17A showed an enrichment for microbiome correlations. Also of interest was that these Tfh gene-bacterial relationships were represented in both adult and young samples.

\section{Discussion}

The $\mathrm{CD} 4^{+} \mathrm{T}$ helper cell population in humans is composed of multiple subsets with clear phenotypic and functional differences, including Th1, Th2, Th17, Th9, Treg, and Tfh. The development of each of the Th cell subsets is driven by lineage-specific transcription factors: T-bet/TXB21 for Th1, GATA3 for Th2, ROR $\alpha / \gamma$ for Th17, FOXO1 for Th9, and FOXP3 for Treg cells. The critical transcription factor for Tfh cells is Bcl6[1, 4, 6, 30]. These various cell types respond to different stimuli including antigenic challenge from pathogens and support development and maturation of both B and T cell effector functions [31]. Cell subsets with similar features have been identified in most mammals, particularly related to rodent and nonhuman primate disease models. Using a nonhuman primate model of ligature-induced periodontitis, we have shown aging effects on the genomic footprints of various $\mathrm{T}$ cell subsets in gingival tissues with health, progressing disease lesions, and clinical resolution [19]. Additionally, we have identified major gingival responses in the model, particularly later in disease progression, that reflected adaptive immune system triggering with substantial expression of rearranged Ig genes $[26,27,32]$. Of particular note for this study is that chronically inflamed tissues commonly accumulate lymphoid aggregates that would facilitate localized T-B cell interactions. These lymphocytes that migrate into chronically inflamed tissues can form ectopic lymphoid structures with functional germinal centers and have been termed tertiary lymphoid structures (TLS) [14] that demonstrate prototypical T follicular helper (Tfh) cells with elevated expression of Bcl6, CXCR5, PD1, and ICOS[1]. Understanding the range of T cell populations that can provide help to B cells within these chronically inflamed tissues is essential to a mechanistic clarification of the biology of these inflammatory conditions. More specifically within the gingival tissues of periodontitis lesions, Tfh cells may be important for effective responses to pathogens, as well as commensal bacteria, as they are required for antibody response quality.

This study targeted gene expression of an array of Tfh genes including various transcription factors (BCL6, STAT3, ASCL2, BATF, c-MAF, IRF4)[4, 33-37]. BCL6 was increased in gingival tissues from both age groups, particularly later in disease progression and the pattern differed from other $\mathrm{T}$ helper cell regulatory transcription factors. A major role for high Bcl6 levels is directing the positioning of Tfh cells to B cells through upregulation of CXCR5 and downregulation of CCR7, PSGL1, and EBI2 [13]. Generally low levels of these 3 genes were also noted with disease in tissues from both age groups. IRF4 and BATF are both essential transcription factors for early Tfh differentiation[10, 35, 36]. BATF showed minimal change with disease; however, IRF4 increased significantly in both age groups, particularly at the disease progression sampling points. STAT3 is also crucial for Tfh cells role for inducing GC B-cells. Both STAT3 and IRF4 are also critical transcription factors for production of IL-21 that impacts Tfh cell development[34, 38, 39]. STAT3 was increased at disease initiation and progression, and decreased to baseline levels with resolution.

IL-21, IL-12, IL-23, and TGFß are all required for development of Tfh cells and IL-21 plays a critical role in regulating Ig production and GC formation. It also acts in an autocrine fashion for Tfh cell development [40-42]. While IL-21 is induced by IL-6 through the STAT3 transcription factor, the mRNA for this cytokine showed minimal change with disease in young animals but was significantly increased later in disease in the adult animals. IL-12 induces generation of Tfh cells from naïve CD4 ${ }^{+} \mathrm{T}$ cells $[43,44]$, while IL-23 has been 
suggested to contribute to the induction of autoantibody formation in various inflammatory diseases [45-47]. Neither of these factors were altered at the mRNA level in this chronic inflammatory disease model.

Tfh cells show elevated expression of various cytokine (IL6R, IL21R) and chemokine (CXCR4, CXCR5) receptors $[7,10]$. Tfh cells co-localize with B cells by Bcl6 up-regulating CXCR5 and down-regulating CCR7, with CXCR5 enhancing migration of Tfh to B cell regions via interaction with its ligand CXCL13 [1, 9, 15, 31]. We identified significant increases in CXCR5 in both adult and young tissues that increased later in disease and actually remained elevated in the resolution samples, while no change in expression of CCR7 was seen. CXCR4 was also elevated, but appeared earlier in the disease process and then dropped to baseline in resolution samples. ICOS is expressed on activated T cells and its ligand, ICOS-L (B7h) is broadly expressed on APCs, non-lymphoid tissues and is constitutively expressed on B cells[48, 49]. The interaction of ICOS/ICOS-L is critical for increases in antigen-specific CXCR $5^{+} \mathrm{PD} 1^{+}$Tfh cells and GC-B cells and has been shown to be dependent on IL-21, IL-6 and STAT3[12]. ICOS is also important for IL-21 and IL-4 production under control of SLAM molecules [39]. Importantly, ICOS engagement induces c-Maf expression that supports Tfh formation and maintenance of these cells through production of IL-21[37]. ICOS mRNA is increased later in disease in both adult and young animals, although levels of c-Maf mRNA were generally unaffected.

CD40LG expressed on Tfh cells binds to the CD40 receptor on B cells as a major co-stimulatory molecule [50, 51]. This interaction induces expression of CD25 on the B cells that helps control B cell receptor expression, and represents a phenotype of memory B-cell population with improved proliferative and antigen-presenting capacity. We noted that increases in message for CD40LG was a response change with disease primarily in adult animals. IL-6 production is generally felt to arise from follicular dendritic cells for Tfh efficient cell responses[5, 52], albeit dendritic cell numbers and functions have also been reported within periodontitis lesions [53-55]. Furthermore, T cell-specific deletion of the IL-6 receptor $\alpha$ chain (IL6RA) results in impaired $\mathrm{T}$ cell responses in vivo, including a defect in Tfh functions [56]. The periodontitis model showed increases in IL6RA only in adult disease, thus representing one of the varied differences in gene expression related to Tfh functions based upon age. PD1 on Tfh cells binds to PD1L on B cells and engages APCs via TCR signaling that provides transient help to B cells, but not Tfh proliferation[57]. It has been found that elevated PD1 actually limits Tfh proliferation through dampening TCR signaling. However, no changes in the mRNA for this important Tfh receptor was observed in this disease model.

CD84/SLAM5 and CD150/SLAM molecules have been identified on GC Tfh cells (Calpe 2008). Through the activity of signaling lymphocyte activation molecule (SLAM)-associated protein (SAP/SH2D1A) adaptor molecule enables these receptors to have a critical role in B-cell help and antibody class switching related to Tfh development [58-60]. While the Tfh interactions with DCs appear primarily via integrin and TCR receptors, the sustained phase of Tfh interaction with B cells requires SAP. The SLAM receptors on Tfh cells are engaged in prolonged T-B cell contact and optimal Tfh functions [61, 62]. In samples from both adult and young animals, both CD84 and CD150 genes were up-regulated later in the disease process, consistent with an increased role of Tfh cells and adaptive immune responses at this stage of the periodontal lesions.

Sphingosine-1-phosphate (S1P) is a biologically active metabolite of plasma-membrane sphingolipids that is essential for immune-cell trafficking. Its receptor, S1PR1, signaling is a crucial factor in regulating naive Tfhcells egress from draining lymph nodes [63]. The gene expression for this receptor was significantly increased in gingival tissues of both adults and young animals primarily at early time points in the disease process and then returned to baseline levels in resolution samples. This is consistent with a role in localization of Tfh cell help within the developing periodontal lesion that could contribute to enhancing control over the host-bacterial interactions during disease. Additionally, CD10/MME/neprilysin is a marker of immature T and B cells and GC B cells and is virtually absent on circulating mature T cells [64]. However, it is not only expressed on Tfh cells, but was substantially increased early in the disease process, again potentially related to the localization and communication of Th cells in this inflammatory environment.

Finally, a number of biomolecules associated with Tfh cells specifically down-regulate functions associated with T-B cell interactions. CD200 is a glycoprotein surface molecule that is a member of the immunoglobulin 
superfamily and is expressed on B cells and a subset of $\mathrm{T}$ cells, including Tfh particularly in reactive lymphoid tissues $[65,66]$. It binds to CD200R1, which is an inhibitory receptor for inflammatory mediator production from lymphoid cells. Of interest is the significant decrease in this molecule across all disease time points, thus potentially contributing to less control over the developing chronic inflammatory lesion. Similarly, B- and Tlymphocyte attenuator (BTLA, eg. CD272) is a co-inhibitory receptor also belonging to the immunoglobulin superfamily. It is induced during $\mathrm{T}$ cell activation except on Th2 cells [67]. BTLA interacts with the B7H4 co-stimulatory molecule and inhibits $\mathrm{T}$ cell effector functions. In the nonhuman primate gingival tissues BTLA mRNA levels were significantly increased during disease, which may contribute to overall regulation of the immune responses occurring through Tfh help in the gingival tissues. SELPLG encodes for P-selectin glycoprotein ligand-1 (PSGL-1) that is an adhesion and regulatory molecule involved in immune cell trafficking. However, PSGL-1 has emerged as an important checkpoint in negatively regulating Tfh cell function during adaptive immune responses $[5,9]$. As with BTLA, this gene was significantly up-regulated only during disease that might be predicted to be an important portion of the $\mathrm{T}$ cell regulatory environment in the gingival tissues. It has been reported that IL-2 signaling is another potent inhibitor of Tfh cell differentiation [68]. While IL2RA mRNA levels increased significantly with disease in both adults and young samples, generally, IL-2 gene levels were generally low in the gingival tissues. STAT5 is the downstream target of IL-2 engagement of IL-2RA. Thus, although STAT5 would also represses Tfh differentiation there was no change in STAT5 mRNA with disease in either age group.

As we noted these substantive changes in the footprint of genes related to Tfh functions, it must be recognized that these changes result from alterations in the quality and quantity of the microbiome at disease sites and the resulting clinical changes reflecting the disease process. The current paradigm in periodontitis is the transition from a healthy symbiotic microbiome to a dysbiotic microbiome at disease sites [69-73]. This dysbiosis reflects altered biology of both pathogens and commensals in the disease ecology, the summation of which drives host response changes, dysregulates these responses, and triggers tissue changes of periodontitis. However, a more detailed profile of the bacteria and/or bacterial complexes related to specific changes in host responses in situ remains to be elucidated. Studies of human gingival transcriptomes and associated targeted bacteria have identified relationships of changes in the expression of specific genes with altered levels of some microbes as indicative of disease versus health; however, these are "point-in-time" samples with minimal knowledge regarding the true dynamics of the lesion development [74-78]. Thus, using this nonhuman primate model of progressing disease, we examined the interrelationship of the microbiome with the pattern of gene expression to discern the potential that certain bacteria or complexes of bacteria afforded a high level of "control" over the gene expression profiles. Multiple outcomes of this analysis were detected. First, there were a finite number of complexes of bacteria that strongly correlated with the Tfh gene expression. The complexes differed in health, disease and resolution sites, as well as showing unique differences in the adult and young microbiomes. Moreover, these complexes were not simply composed of only bacteria historically considered as periodontopathogens versus oral commensal bacteria, but generally were mixtures of these types of microorganisms. Secondly, certain of these complexes were significantly correlated with multiple genes that generally were either positively or negatively correlated with the panel of Tfh genes (eg. Tfh-H1A, Tfh-R2A, Tfh-R4A, Tfh-D1Y). Finally, while we could estimate statistical significance of specific correlations of complexes of bacteria with specific Tfh genes, a systems approach to the relationship of bacterial abundance to gene expression levels provided a visualization of broad correlations between specific bacterial complexes and panels of the Tfh genes. Of note, the same bacterial complex demonstrated positive and negative correlation patterns with different groups of genes within the Tfh footprint.

Thus, Tfh cell functions are mediated through the production of cytokines that also promote B-cell survival and antibody production, and through the engagement of T-APC and T-B cell co-stimulatory molecules (eg. TCR, ICOS, CD40LG, PD1,) and other receptors (eg. SLAM, CXCR5, etc.), which favor these strong interactions leading to successful and mature B-cell immunoglobulin responses. Coupled with existing data in humans [79-85] and nonhuman primates [20, 86, 87] detailing local and systemic antibody production that can be enhanced by immunization, supports that localized Tfh functions in gingival tissues may provide an important adaptive immune regulatory mechanism for controlling the progression and magnitude of disease. 


\section{Acknowledgement}

This work was supported by National Institute of Health grant P20GM103538. We express our gratitude to the Caribbean Primate Research Center (CPRC) supported by grant P40RR03640, and the Center for Oral Health Research in the College of Dentistry at the University of Kentucky. We also thank the Microarray Core of University Kentucky for their invaluable technical assistance, and Dr. A. Stromberg for initial normalization of the microarray data. The authors acknowledge no conflict of interest with the content of this report.

\section{Author Contributions}

JLE and OAG were responsible for the design, conduct of the experiment, clinical data and sample collection, interpretation of the data, and preparation of the manuscript. SSK was responsible for preparation of the gingival tissue mRNA and bacterial DNA for analysis. LO contributed to the clinical measures, and JGM contributed to the animal husbandry and conduct of the experiment.

\section{References}

1. Crotty S. T Follicular Helper Cell Biology: A Decade of Discovery and Diseases. Immunity 2019; 50 :1132-48.

2. Crotty S. T follicular helper cell differentiation, function, and roles in disease. Immunity 2014; 41 :529-42.

3. Crotty S. Follicular helper CD4 T cells (TFH). Annual review of immunology 2011; 29 :621-63.

4. Hatzi K, Nance JP, Kroenke MA, Bothwell M, Haddad EK, Melnick A, Crotty S. BCL6 orchestrates Tfh cell differentiation via multiple distinct mechanisms. The Journal of experimental medicine 2015;212 :539-53.

5. Poholek AC, Hansen K, Hernandez SG, Eto D, Chandele A, Weinstein JS, Dong X, Odegard JM, Kaech $\mathrm{SM}$, Dent AL, Crotty S, Craft J. In vivo regulation of Bcl6 and T follicular helper cell development. J Immunol 2010; $185: 313-26$.

6. Nurieva RI, Chung Y, Martinez GJ, Yang XO, Tanaka S, Matskevitch TD, Wang YH, Dong C. Bcl6 mediates the development of $\mathrm{T}$ follicular helper cells. Science 2009; $325: 1001-5$.

7. Yu D, Rao S, Tsai LM, Lee SK, He Y, Sutcliffe EL, Srivastava M, Linterman M, Zheng L, Simpson N, Ellyard JI, Parish IA, Ma CS, Li QJ, Parish CR, Mackay CR, Vinuesa CG. The transcriptional repressor Bcl-6 directs T follicular helper cell lineage commitment. Immunity 2009; 31 :457-68.

8. Qin L, Waseem TC, Sahoo A, Bieerkehazhi S, Zhou H, Galkina EV, Nurieva R. Insights Into the Molecular Mechanisms of T Follicular Helper-Mediated Immunity and Pathology. Frontiers in immunology 2018; 9 :1884.

9. Vinuesa CG, Linterman MA, Yu D, MacLennan IC. Follicular Helper T Cells. Annual review of immunology $2016 ; 34: 335-68$.

10. Schmitt N, Liu Y, Bentebibel SE, Ueno H. Molecular Mechanisms Regulating T Helper 1 versus $\mathrm{T}$ Follicular Helper Cell Differentiation in Humans. Cell reports 2016; 16 :1082-95.

11. Morita R, Schmitt N, Bentebibel SE, Ranganathan R, Bourdery L, Zurawski G, Foucat E, Dullaers M, Oh S, Sabzghabaei N, Lavecchio EM, Punaro M, Pascual V, Banchereau J, Ueno H. Human blood CXCR5(+)CD4(+) T cells are counterparts of $\mathrm{T}$ follicular cells and contain specific subsets that differentially support antibody secretion. Immunity $2011 ; 34: 108-21$.

12. Akiba H, Takeda K, Kojima Y, Usui Y, Harada N, Yamazaki T, Ma J, Tezuka K, Yagita H, Okumura $\mathrm{K}$. The role of ICOS in the CXCR5+ follicular B helper T cell maintenance in vivo. J Immunol 2005;175 $: 2340-8$. 
13. Rao DA. T Cells That Help B Cells in Chronically Inflamed Tissues. Frontiers in immunology 2018; 9 :1924.

14. Cicalese MP, Salek-Ardakani S, Fousteri G. Editorial: Follicular Helper T Cells in Immunity and Autoimmunity. Frontiers in immunology 2020; $11: 1042$.

15. Deng J, Wei Y, Fonseca VR, Graca L, Yu D. T follicular helper cells and T follicular regulatory cells in rheumatic diseases. Nature reviews Rheumatology 2019; 15 :475-90.

16. Gonzalez OA, Novak MJ, Kirakodu S, Stromberg AJ, Shen S, Orraca L, Gonzalez-Martinez J, Ebersole JL. Effects of aging on apoptosis gene expression in oral mucosal tissues. Apoptosis : an international journal on programmed cell death $2013 ; 18: 249-59$.

17. Gonzalez OA, Stromberg AJ, Huggins PM, Gonzalez-Martinez J, Novak MJ, Ebersole JL. Apoptotic genes are differentially expressed in aged gingival tissue. J Dent Res 2011; 90 :880-6.

18. Ebersole JL, Steffen MJ, Reynolds MA, Branch-Mays GL, Dawson DR, Novak KF, Gunsolley JC, Mattison JA, Ingram DK, Novak MJ. Differential gender effects of a reduced-calorie diet on systemic inflammatory and immune parameters in nonhuman primates. J Periodontal Res 2008;43 :500-7.

19. Ebersole JL, Kirakodu S, Novak MJ, Stromberg AJ, Shen S, Orraca L, Gonzalez-Martinez J, Burgos A, Gonzalez OA. Cytokine gene expression profiles during initiation, progression and resolution of periodontitis. J Clin Periodontol 2014.

20. Ebersole JL, Steffen MJ, Gonzalez-Martinez J, Novak MJ. Effects of age and oral disease on systemic inflammatory and immune parameters in nonhuman primates. Clin Vaccine Immunol 2008;15 :1067-75.

21. Kirakodu S, Chen J, Gonzalez Martinez J, Gonzalez OA, Ebersole J. Microbiome Profiles of LigatureInduced Periodontitis in Nonhuman Primates Across the Lifespan. Infect Immun 2019.

22. Kozich JJ, Westcott SL, Baxter NT, Highlander SK, Schloss PD. Development of a dual-index sequencing strategy and curation pipeline for analyzing amplicon sequence data on the MiSeq Illumina sequencing platform. Appl Environ Microbiol 2013; 79 :5112-20.

23. Schloss PD, Westcott SL, Ryabin T, Hall JR, Hartmann M, Hollister EB, Lesniewski RA, Oakley BB, Parks DH, Robinson CJ, Sahl JW, Stres B, Thallinger GG, Van Horn DJ, Weber CF. Introducing mothur: open-source, platform-independent, community-supported software for describing and comparing microbial communities. Appl Environ Microbiol 2009;75 :7537-41.

24. Edgar RC, Haas BJ, Clemente JC, Quince C, Knight R. UCHIME improves sensitivity and speed of chimera detection. Bioinformatics 2011;27:2194-200.

25. Kirakodu S, Chen J, Gonzalez Martinez J, Gonzalez OA, Ebersole J. Microbiome Profiles of LigatureInduced Periodontitis in Nonhuman Primates across the Life Span. Infect Immun 2019; 87.

26. Gonzalez OA, Novak MJ, Kirakodu S, Stromberg A, Nagarajan R, Huang CB, Chen KC, Orraca L, Martinez-Gonzalez J, Ebersole JL. Differential Gene Expression Profiles Reflecting Macrophage Polarization in Aging and Periodontitis Gingival Tissues. Immunological investigations 2015; 44 :643-64.

27. Gonzalez OA, Novak MJ, Kirakodu S, Orraca L, Chen KC, Stromberg A, Gonzalez-Martinez J, Ebersole JL. Comparative analysis of gingival tissue antigen presentation pathways in ageing and periodontitis.J Clin Periodontol 2014; 41 :327-39.

28. Gonzalez OA, John Novak M, Kirakodu S, Stromberg AJ, Shen S, Orraca L, Gonzalez-Martinez J, Ebersole JL. Effects of aging on apoptosis gene expression in oral mucosal tissues. Apoptosis 2013;18:24959.

29. Meka A, Bakthavatchalu V, Sathishkumar S, Lopez MC, Verma RK, Wallet SM, Bhattacharyya I, Boyce 
BF, Handfield M, Lamont RJ, Baker HV, Ebersole JL, Kesavalu L. Porphyromonas gingivalis infectioninduced tissue and bone transcriptional profiles. Mol Oral Microbiol 2010; 25 :61-74.

30. Choi J, Diao H, Faliti CE, Truong J, Rossi M, Belanger S, Yu B, Goldrath AW, Pipkin ME, Crotty $\mathrm{S}$. Bcl-6 is the nexus transcription factor of $\mathrm{T}$ follicular helper cells via repressor-of-repressor circuits. Nat Immunol 2020; $21: 777-89$.

31. Nurieva RI, Chung Y. Understanding the development and function of T follicular helper cells. Cellular \& molecular immunology 2010;7 :190-7.

32. Ebersole JL, Kirakodu SS, Novak MJ, Orraca L, Martinez JG, Cunningham LL, Thomas MV, Stromberg A, Pandruvada SN, Gonzalez OA. Transcriptome Analysis of B Cell Immune Functions in Periodontitis: Mucosal Tissue Responses to the Oral Microbiome in Aging.Frontiers in immunology 2016; 7 :272.

33. Liu X, Chen X, Zhong B, Wang A, Wang X, Chu F, Nurieva RI, Yan X, Chen P, van der Flier LG, Nakatsukasa H, Neelapu SS, Chen W, Clevers H, Tian Q, Qi H, Wei L, Dong C. Transcription factor achaete-scute homologue 2 initiates follicular T-helper-cell development.Nature 2014; 507 :513-8.

34. Ma CS, Avery DT, Chan A, Batten M, Bustamante J, Boisson-Dupuis S, Arkwright PD, Kreins AY, Averbuch D, Engelhard D, Magdorf K, Kilic SS, Minegishi Y, Nonoyama S, French MA, Choo S, Smart JM, Peake J, Wong M, Gray P, Cook MC, Fulcher DA, Casanova JL, Deenick EK, Tangye SG. Functional STAT3 deficiency compromises the generation of human T follicular helper cells. Blood 2012; 119 :3997-4008.

35. Betz BC, Jordan-Williams KL, Wang C, Kang SG, Liao J, Logan MR, Kim CH, Taparowsky EJ. Batf coordinates multiple aspects of $\mathrm{B}$ and $\mathrm{T}$ cell function required for normal antibody responses. The Journal of experimental medicine 2010; $207: 933-42$.

36. Bollig N, Brustle A, Kellner K, Ackermann W, Abass E, Raifer H, Camara B, Brendel C, Giel G, Bothur E, Huber M, Paul C, Elli A, Kroczek RA, Nurieva R, Dong C, Jacob R, Mak TW, Lohoff M. Transcription factor IRF4 determines germinal center formation through follicular T-helper cell differentiation. Proc Natl Acad Sci U S A 2012;109:8664-9.

37. Bauquet AT, Jin H, Paterson AM, Mitsdoerffer M, Ho IC, Sharpe AH, Kuchroo VK. The costimulatory molecule ICOS regulates the expression of c-Maf and IL-21 in the development of follicular T helper cells and TH-17 cells. Nat Immunol 2009; 10 :167-75.

38. Schmitt N, Liu Y, Bentebibel SE, Munagala I, Bourdery L, Venuprasad K, Banchereau J, Ueno H. The cytokine TGF-beta co-opts signaling via STAT3-STAT4 to promote the differentiation of human TFH cells.Nat Immunol 2014; $15: 856-65$.

39. Nurieva RI, Chung Y, Hwang D, Yang XO, Kang HS, Ma L, Wang YH, Watowich SS, Jetten AM, Tian Q, Dong C. Generation of T follicular helper cells is mediated by interleukin-21 but independent of T helper 1, 2, or 17 cell lineages. Immunity 2008; $29: 138-49$.

40. Gong F, Zheng T, Zhou P. T Follicular Helper Cell Subsets and the Associated Cytokine IL-21 in the Pathogenesis and Therapy of Asthma. Frontiers in immunology 2019; 10 :2918.

41. Tangye SG. Advances in IL-21 biology - enhancing our understanding of human disease. Curr Opin Immunol 2015; $34: 107-15$.

42. Spolski R, Leonard WJ. Interleukin-21: a double-edged sword with therapeutic potential. Nat Rev Drug Discov 2014;13 :379-95.

43. Schmitt N, Bustamante J, Bourdery L, Bentebibel SE, Boisson-Dupuis S, Hamlin F, Tran MV, Blankenship D, Pascual V, Savino DA, Banchereau J, Casanova JL, Ueno H. IL-12 receptor beta1 deficiency alters in vivo T follicular helper cell response in humans. Blood 2013;121 :3375-85.

44. Ma CS, Suryani S, Avery DT, Chan A, Nanan R, Santner-Nanan B, Deenick EK, Tangye SG. Early commitment of naive human $\mathrm{CD} 4(+) \mathrm{T}$ cells to the $\mathrm{T}$ follicular helper $(\mathrm{T}(\mathrm{FH}))$ cell lineage is induced by 


\section{IL-12.Immunol Cell Biol 2009; 87 :590-600.}

45. Menegat JS, Lira-Junior R, Siqueira MA, Brito F, Carvalho AT, Fischer RG, Figueredo CM. Cytokine expression in gingival and intestinal tissues of patients with periodontitis and inflammatory bowel disease: An exploratory study. Arch Oral Biol 2016; 66 :141-6.

46. Jain R, Chen Y, Kanno Y, Joyce-Shaikh B, Vahedi G, Hirahara K, Blumenschein WM, Sukumar S, Haines CJ, Sadekova S, McClanahan TK, McGeachy MJ, O'Shea JJ, Cua DJ. Interleukin-23-Induced Transcription Factor Blimp-1 Promotes Pathogenicity of T Helper 17 Cells.Immunity 2016; $44: 131-42$.

47. Wendling D, Abbas W, Godfrin-Valnet M, Kumar A, Guillot X, Khan KA, Vidon C, Coquard L, Toussirot E, Prati C, Herbein G. Dysregulated serum IL-23 and SIRT1 activity in peripheral blood mononuclear cells of patients with rheumatoid arthritis. PLoS One 2015;10 :e0119981.

48. Uwadiae FI, Pyle CJ, Walker SA, Lloyd CM, Harker JA. Targeting the ICOS/ICOS-L pathway in a mouse model of established allergic asthma disrupts $\mathrm{T}$ follicular helper cell responses and ameliorates disease. Allergy 2019; $74: 650-62$.

49. Choi YS, Kageyama R, Eto D, Escobar TC, Johnston RJ, Monticelli L, Lao C, Crotty S. ICOS receptor instructs $\mathrm{T}$ follicular helper cell versus effector cell differentiation via induction of the transcriptional repressor Bcl6. Immunity 2011; 34 :932-46.

50. Grewal IS, Flavell RA. The CD40 ligand. At the center of the immune universe? Immunol Res 1997; 16 $: 59-70$.

51. Liu YJ, Grouard G, de Bouteiller O, Banchereau J. Follicular dendritic cells and germinal centers. Int Rev Cytol 1996;166 :139-79.

52. Choi YS, Eto D, Yang JA, Lao C, Crotty S. Cutting edge: STAT1 is required for IL-6-mediated Bcl6 induction for early follicular helper cell differentiation. J Immunol 2013; 190 :3049-53.

53. Meyle J, Chapple I. Molecular aspects of the pathogenesis of periodontitis. Periodontol 2000 2015; 69 $: 7-17$.

54. El-Awady AR, Arce RM, Cutler CW. Dendritic cells: microbial clearance via autophagy and potential immunobiological consequences for periodontal disease. Periodontol 2000 2015; 69 :160-80.

55. Souto GR, Queiroz CM, Jr., Costa FO, Mesquita RA. Relationship between chemokines and dendritic cells in human chronic periodontitis. J Periodontol 2014; 85 :1416-23.

56. Nish SA, Schenten D, Wunderlich FT, Pope SD, Gao Y, Hoshi N, Yu S, Yan X, Lee HK, Pasman L, Brodsky I, Yordy B, Zhao H, Bruning J, Medzhitov R. T cell-intrinsic role of IL-6 signaling in primary and memory responses. eLife 2014; 3 :e01949.

57. Jogdand GM, Mohanty S, Devadas S. Regulators of Tfh Cell Differentiation. Frontiers in immunology $2016 ; 7: 520$.

58. Hu J, Havenar-Daughton C, Crotty S. Modulation of SAP dependent T:B cell interactions as a strategy to improve vaccination. Curr Opin Virol 2013; $3: 363-70$.

59. He J, Tsai LM, Leong YA, Hu X, Ma CS, Chevalier N, Sun X, Vandenberg K, Rockman S, Ding Y, Zhu L, Wei W, Wang C, Karnowski A, Belz GT, Ghali JR, Cook MC, Riminton DS, Veillette A, Schwartzberg PL, Mackay F, Brink R, Tangye SG, Vinuesa CG, Mackay CR, Li Z, Yu D. Circulating precursor CCR7(lo)PD1(hi) CXCR5(+) CD4(+) $\mathrm{T}$ cells indicate Tfh cell activity and promote antibody responses upon antigen reexposure. Immunity $2013 ; 39: 770-81$.

60. Cannons JL, Qi H, Lu KT, Dutta M, Gomez-Rodriguez J, Cheng J, Wakeland EK, Germain RN, Schwartzberg PL. Optimal germinal center responses require a multistage T cell:B cell adhesion process involving integrins, SLAM-associated protein, and CD84. Immunity 2010;32 :253-65. 
61. Biram A, Davidzohn N, Shulman Z. T cell interactions with B cells during germinal center formation, a three-step model.Immunological reviews 2019; $288: 37-48$.

62. Qi H. From SAP-less T cells to helpless B cells and back: dynamic T-B cell interactions underlie germinal center development and function. Immunological reviews 2012; 247 :24-35.

63. Moriyama S, Takahashi N, Green JA, Hori S, Kubo M, Cyster JG, Okada T. Sphingosine-1-phosphate receptor 2 is critical for follicular helper $\mathrm{T}$ cell retention in germinal centers. The Journal of experimental medicine 2014; 211 :1297-305.

64. Ame-Thomas P, Hoeller S, Artchounin C, Misiak J, Braza MS, Jean R, Le Priol J, Monvoisin C, Martin N, Gaulard P, Tarte K. CD10 delineates a subset of human IL-4 producing follicular helper T cells involved in the survival of follicular lymphoma B cells. Blood 2015;125 :2381-5.

65. Chakera A, Bennett SC, Morteau O, Bowness P, Luqmani RA, Cornall RJ. The phenotype of circulating follicular-helper T cells in patients with rheumatoid arthritis defines CD200 as a potential therapeutic target. Clinical \& developmental immunology 2012;2012:948218.

66. Chtanova T, Tangye SG, Newton R, Frank N, Hodge MR, Rolph MS, Mackay CR. T follicular helper cells express a distinctive transcriptional profile, reflecting their role as non-Th1/Th2 effector cells that provide help for B cells. J Immunol 2004; 173 :68-78.

67. M'Hidi H, Thibult ML, Chetaille B, Rey F, Bouadallah R, Nicollas R, Olive D, Xerri L. High expression of the inhibitory receptor BTLA in T-follicular helper cells and in B-cell small lymphocytic lymphoma/chronic lymphocytic leukemia. Am J Clin Pathol 2009;132:589-96.

68. Crotty S. A brief history of T cell help to B cells. Nature reviews Immunology 2015; 15 :185-9.

69. Curtis MA, Diaz PI, Van Dyke TE. The role of the microbiota in periodontal disease. Periodontol 2000 2020; $83: 14-25$.

70. Lamont RJ, Koo H, Hajishengallis G. The oral microbiota: dynamic communities and host interactions. Nat Rev Microbiol 2018;16 :745-59.

71. Mira A, Simon-Soro A, Curtis MA. Role of microbial communities in the pathogenesis of periodontal diseases and caries. J Clin Periodontol 2017; 44 Suppl 18 :S23-S38.

72. Kilian M, Chapple IL, Hannig M, Marsh PD, Meuric V, Pedersen AM, Tonetti MS, Wade WG, Zaura E. The oral microbiome - an update for oral healthcare professionals. British dental journal 2016;221 :657-66.

73. Papapanou PN, Park H, Cheng B, Kokaras A, Paster B, Burkett S, Watson CW, Annavajhala MK, Uhlemann AC, Noble JM. Subgingival microbiome and clinical periodontal status in an elderly cohort:The WHICAP ancillary study of oral health. J Periodontol 2020.

74. Sawle AD, Kebschull M, Demmer RT, Papapanou PN. Identification of Master Regulator Genes in Human Periodontitis. J Dent Res 2016;95:1010-7.

75. Kebschull M, Demmer RT, Grun B, Guarnieri P, Pavlidis P, Papapanou PN. Gingival tissue transcriptomes identify distinct periodontitis phenotypes. J Dent Res 2014; 93 :459-68.

76. Kebschull M, Guarnieri P, Demmer RT, Boulesteix AL, Pavlidis P, Papapanou PN. Molecular differences between chronic and aggressive periodontitis. J Dent Res 2013; 92 :1081-8.

77. Stoecklin-Wasmer C, Guarnieri P, Celenti R, Demmer RT, Kebschull M, Papapanou PN. MicroRNAs and their target genes in gingival tissues. J Dent Res 2012; 91 :934-40.

78. Kebschull M, Papapanou PN. The use of gene arrays in deciphering the pathobiology of periodontal diseases. Methods Mol Biol 2010;666 :385-93. 
79. Schenkein HA, Berry CR, Burmeister JA, Brooks CN, Best AM, Tew JG. Locally produced antiphosphorylcholine and anti-oxidized low-density lipoprotein antibodies in gingival crevicular fluid from aggressive periodontitis patients. J Periodontol 2004; 75 :146-53.

80. Ebersole JL. Humoral immune responses in gingival crevice fluid: local and systemic implications. Periodontol 2000 2003;31:135-66.

81. Darby IB, Mooney J, Kinane DF. Changes in subgingival microflora and humoral immune response following periodontal therapy. J Clin Periodontol 2001; 28 :796-805.

82. Ebersole JL, Cappelli D, Steffen MJ. Antigenic specificity of gingival crevicular fluid antibody to Actinobacillus actinomycetemcomitans. J Dent Res 2000; 79 :1362-70.

83. Mooney J, Kinane DF. Levels of specific immunoglobulin G to Porphyromonas gingivalis in gingival crevicular fluid are related to site disease status. Oral Microbiol Immunol 1997;12 :112-6.

84. Ebersole JL, Cappelli D. Gingival crevicular fluid antibody to Actinobacillus actinomycetemcomitans in periodontal disease. Oral Microbiol Immunol 1994; $9: 335-44$.

85. Kinane DF, Mooney J, MacFarlane TW, McDonald M. Local and systemic antibody response to putative periodontopathogens in patients with chronic periodontitis: correlation with clinical indices. Oral Microbiol Immunol 1993; $8: 65-8$.

86. Ebersole JL, Holt SC, Delaney JE. Acquisition of Oral Microbes and Associated Systemic Responses of Newborn Nonhuman Primates.Clinical and vaccine immunology 2014; 21 :21-8.

87. Ebersole JL, Cappelli D, Holt SC, Singer RE, Filloon T. Gingival crevicular fluid inflammatory mediators and bacteriology of gingivitis in nonhuman primates related to susceptibility to periodontitis. Oral Microbiol Immunol 2000; $15: 19-26$.

Table 1: $\mathrm{T}$ follicular helper cell gene expression targets

\begin{tabular}{lll}
\hline Gene ID & Fxn & Gene ID \\
\hline ASCL2 & Achaete-Scute Family BHLH Transcription Factor 2 & IFNG \\
BATF & Basic Leucine Zipper ATF-Like Transcription Factor & IL2RA \\
BCL6 & BCL6 Transcription Repressor; master transcription factor Tfh & IL4 \\
BTLA & B And T Lymphocyte Associated (CD272) attenuator; Inhibitory receptor on lymphocytes & IL6RA \\
CCR7 & C-C Motif Chemokine Receptor 7 & IL7R \\
CD3D & TCR component; binds MHCI on APCs & IL10 \\
CD3E & TCR component; binds MHCII on APCs & IL12 \\
CD3G & TCR component; binds MHCII on APCs & IL17A \\
CD4 & Essential role in immune responses; subset of T cells & IL17F \\
CD10/MME & Neprilysin; membrane metallopeptidase & IL21 \\
CD40LG & T cell surface ligand; binds CD40 on B cells & IL21R \\
CD57/B3GAT1 & Biosynthesis of L2/HNK-1 carbohydrate epitope on glycoproteins & IL23 \\
CD84/SLAMF5 & Signaling lymphocyte activation molecule (SLAM) family & IRF4 \\
CD150/SLAMF1 & Signaling lymphocyte activation molecule (SLAM) family & c-maf/MA \\
CD200 & OX-2; Costimulates T-cell proliferation & PD1/PDC \\
CXCL13 & C-X-C Motif Chemokine Ligand 13 & RORA \\
CXCR4 & C-X-C Motif Chemokine Receptor 4 & RORC \\
CXCR5 & C-X-C Motif Chemokine Receptor 5 & S1PR1 \\
EBI2/GPR183 & G Protein-Coupled Receptor 183 & SELPLG/ \\
FOXP3 & Forkhead Box P3; master transcription factor Treg & SH2D1A/ \\
FOXO1 & Forkhead Box O1; master transcription factor Th9 & STAT3 \\
GATA3 & GATA Binding Protein 3; master transcription factor Th2 & TGFB1
\end{tabular}


Gene ID

ICOS
Fxn

Inducible T Cell Costimulator; binds ICOSL on B cells
Gene ID

T-bet/TX

Table 2: Significant correlations of microbiome OTU complexes with genes related to Tfh phenotype and functions. Blue denotes significant positive correlation and red denotes a significant negative correlation. 
Healthfh- Fretibacteriutm +

H1A sp.

361

Chlo-

roflexi_-

[G-

1]

sp.

439

Fretibac-

terium

fas-

tid-

io-

sum

363

Tre-

ponema

sp.

246

Pep-

tostrep-

to-

coc-

caceae_-

[XIII]_-

un-

clas-

si-

fied

Por-

phy-

romonas

gin-

gi-

valis

619

A.

acti-

no-

mycetem-

comi-

tans

531

Fil-

i-

fac-

tor

alocis

539 
Tfh- Selenomonas_- $+\quad+\quad+$

$\mathrm{H} 2 \mathrm{~A}$ un-

clas-

si-

fied

Lep-

totrichia_-

un-

clas-

si-

fied

Veil-

lonella

un-

clas-

si-

fied

Pre-

votella

un-

clas-

si-

fied

Pre-

votella

enoeca

600

Ag-

gre-

gat-

i-

bac-

ter

un-

clas-

si-

fied

Neis-

se-

ria

oralis

014 


Tfh- Bacteria_- $++_{+}+$

H3A un-

clas-

si-

fied

Tre-

ponema

un-

clas-

si-

fied

Pyra-

mi-

dobac-

ter

pis-

colens

357

Se-

lenomonas

sputi-

gena

151

Veil-

lonel-

laceae_-

[G-

1]

sp.

155

Pre-

votella

in-

ter-

me-

dia

643

Pre-

votella

sp.

820

Tre-

ponema

mal-

tophilum

664 
Tfh- Eubacterium +

$\mathrm{H} 4 \mathrm{~A}$ in-

fir-

mum

105

Strep-

to-

coc-

cus

Pre-

votella

sp.

526

Gemella

mor-

bil-

lo-

rum

046

Por-

phy-

romonas

sp.

279

Moraxella catarrhalis

833

Haemophilus

sp.

035 
Disea\$eh- Leptotrichia_- $+\quad+\quad+\quad+$

D1A un-

clas-

si-

fied

Veil-

lonella

un-

clas-

si-

fied

Se-

lenomonas

sputi-

gena

151

Fretibac-

terium

un-

clas-

si-

fied

Tre-

ponema

mal-

tophilum

664

$\mathrm{Fu}-$

sobac-

terium

sp.

203

Veil-

lonel-

laceae_-

[G-

1]

sp.

155

Pre-

votella

sp.

317

Se-

lenomonas

unclassified 
c-

Categфb Complew3GIL6RACD40RD3DIL12 IL21RCD84CD15@D4 CD57CD10IL17ACXCRBTLAmaf IL21 IFNGPD1

Tfh- Fretibacteriutm

$+$

$+$

D2A fas-

tid-

io-

sum

363

Pep-

tostrep-

to-

coc-

caceae_-

[XIII]_-

un-

clas-

si-

fied

Tre-

ponema

un-

clas-

si-

fied

Fretibac-

terium

sp.

361

Tre-

ponema

den-

ti-

cola

584

Pre-

votella

enoeca

600 
c-

Categфb Complew3GIL6RACD40RD3DIL12 IL21RCD84CD15@D4 CD57CD10IL17ACXCRBTLAmaf IL21 IFNGPD1

Tfh- Haemophilus

D3A sp.

035

Strep-

to-

coc-

cus

sp.

058

Por-

phy-

romonas

sp.

279

Moraxella

catarrhalis

833

Gemella

mor-

bil-

lo-

rum

046

Porphyromonadaceae

Tfh- Porphyromołas

D4A en-

dodon-

talis

273

Por-

phy-

romonas

gin-

gi-

valis

619

Tre-

ponema

sp.

246

$\mathrm{Fu}-$

sobac-

terium

unclassified 
ResolfffionStreptococcus_-

R1A un-

clas-

si-

fied

Veil-

lonella

parvula

161

Haemophilus

sp.

035

Pre-

votella

sp.

311

Megas-

phaera

mi-

cronu-

ci-

formis

122

Pre-

votella

den-

ti-

cola

291

Pre-

votella

un-

clas-

si-

fied

Veil-

lonella

un-

clas-

si-

fied

Veil-

lonella

dis-

par

160 
c-

Categфb Complew3GIL6RACD40RD3DIL12 IL21RCD84CD15@D4 CD57CD10IL17ACXCRBTLAmaf IL21 IFNGPD1

Tfh- Streptococcus + + + + + +

$\mathrm{R} 2 \mathrm{~A}$ sp.

058

Pre-

votella

sp.

317

Catonella

morbi

165

Lep-

totrichia_-

un-

clas-

si-

fied

Se-

lenomonas_-

un-

clas-

si-

fied

Tre-

ponema

socran-

skii

769

Se-

lenomonas

unclassified 
Tfh- Fretibacterium

R3A un-

clas-

si-

fied

Gemella

mor-

bil-

lo-

rum

046

Cap-

no-

cy-

tophaga_-

un-

clas-

si-

fied

Por-

phy-

romonas

sp.

279

$\mathrm{Fu}-$

sobac-

terium

unclassified 
CategळD Complex3GL6RACD40RD3DIL12 IL21RCD84CD15@D4 CD57CD10IL17ACXCRBTLAmaf IL21 IFNGPD1

Tfh- Fusobacterium $+t_{+}+$

R4A sp.

203

Chlo-

roflexi_-

[G-

1]

sp.

439

Pre-

votella

sp.

526

$\mathrm{Eu}-$

bac-

terium

in-

fir-

mum

105

A.

acti-

no-

mycetem-

comi-

tans

531

Tre-

ponema

un-

clas-

si-

fied

Tre-

ponema

den-

ti-

cola

584 
Tfh- Filifactor

R5A alocis

539

Pep-

tostrep-

to-

coc-

caceae_-

[XIII]_-

un-

clas-

si-

fied

Fretibac-

terium

sp.

361

Por-

phy-

romonas

gin-

gi-

valis

619

Por-

phy-

romonas

en-

dodon-

talis

273

Fretibac-

terium

fas-

tid-

io-

sum

363

Desul-

fob-

ul-

bus

sp.

041 
Healthfh- Gemella

H1Y mor-

bil-

lo-

rum

046

$\mathrm{Fu}-$

sobac-

terium_-

un-

clas-

si-

fied

Fretibac-

terium_-

un-

clas-

si-

fied

Fretibac-

terium

sp.

361

A.

acti-

no-

mycetem-

comi-

tans

531

Fretibac-

terium

fas-

tid-

io-

sum

363 
Tfh- Prevotella + $\mathrm{H} 2 \mathrm{Y}$ in-

ter-

me-

dia

643

Tre-

ponema

den-

ti-

cola

584

Pre-

votella

enoeca

600

Tre-

ponema

sp.

246

Tre-

ponema_-

un-

clas-

si-

fied

Tre-

ponema

mal-

tophilum

664

Pre-

votella

sp.

313 
Tfh- Bacteria_-

H3Y un-

clas-

si-

fied

Veil-

lonella

parvula

161

Pre-

votella

sp.

526

Chlo-

roflexi_-

[G-

1]sp.

439

Pre-

votella

sp.

820

Disea\$€h- Bacterøidetes_-

D1Y un-

clas-

si-

fied

Fretibac-

terium_-

un-

clas-

si-

fied

Pep-

tostrep-

to-

coc-

caceae_-

[XIII]_-

un-

clas-

si-

fied

Tre-

ponema

sp.

246 
Tfh- Bacteroidetes_- +

D2Y un-

clas-

si-

fied

Fretibac-

terium_-

un-

clas-

si-

fied

Pre-

votella

fusca

782

Tre-

ponema_-

un-

clas-

si-

fied

Catonella

morbi

165

Tre-

ponema

den-

ti-

cola

584

Pre-

votella

sp.

304

Por-

phy-

romonas

gin-

gi-

valis

619 
Tfh- Haemophilus

D3Y sp.

035

SR1_-

G-

1]sp.

345

Pre-

votella

sp.

317

Cap-

no-

cy-

tophaga_-

un-

clas-

si-

fied

Por-

phy-

romonas

sp.

279

Pre-

votella

sp.

526 
Tfh- Bacteroidetes_-

D4Y un-

clas-

si-

fied

Fretibac-

terium_-

un-

clas-

si-

fied

Pre-

votella

fusca

782

Tre-

ponema_-

un-

clas-

si-

fied

Tre-

ponema

den-

ti-

cola

584

Pre-

votella

sp.

304

Por-

phy-

romonas

gin-

gi-

valis

619

Fretibac-

terium

fas-

tid-

io-

sum

363

Chlo-

roflexi_-

[G-

1]sp.

439

Bac-

te-

ria_-

un-

clas-

si-

fied 
Tfh- Gemella

R5Y mor-

bil-

lo-

rum

046

Pas-

teurel-

laceae_-

un-

clas-

si-

fied

Strep-

to-

coc-

cus

sp.

058

Fretibac-

terium

sp.

361

Fil-

i-

fac-

tor

alocis

539 
ResolnflionPrevotella

R1Y sp. 304

Fretibac-

terium_-

un-

clas-

si-

fied

Fil-

i-

fac-

tor

alocis

539

Fretibac-

terium

fas-

tid-

io-

sum

363

Chlo-

roflexi_-

[G-

1]sp.

439

Fretibac-

terium

sp.

361

Lep-

totrichia

sp.

223

Veil-

lonel-

laceae_-

G-

1] _-

un-

clas-

si-

fied

Eu-

bac-

terium_-

[XI][G-

1]infirmum

105

Por-

phy-

romonas

gin-

gi-

valis

619 


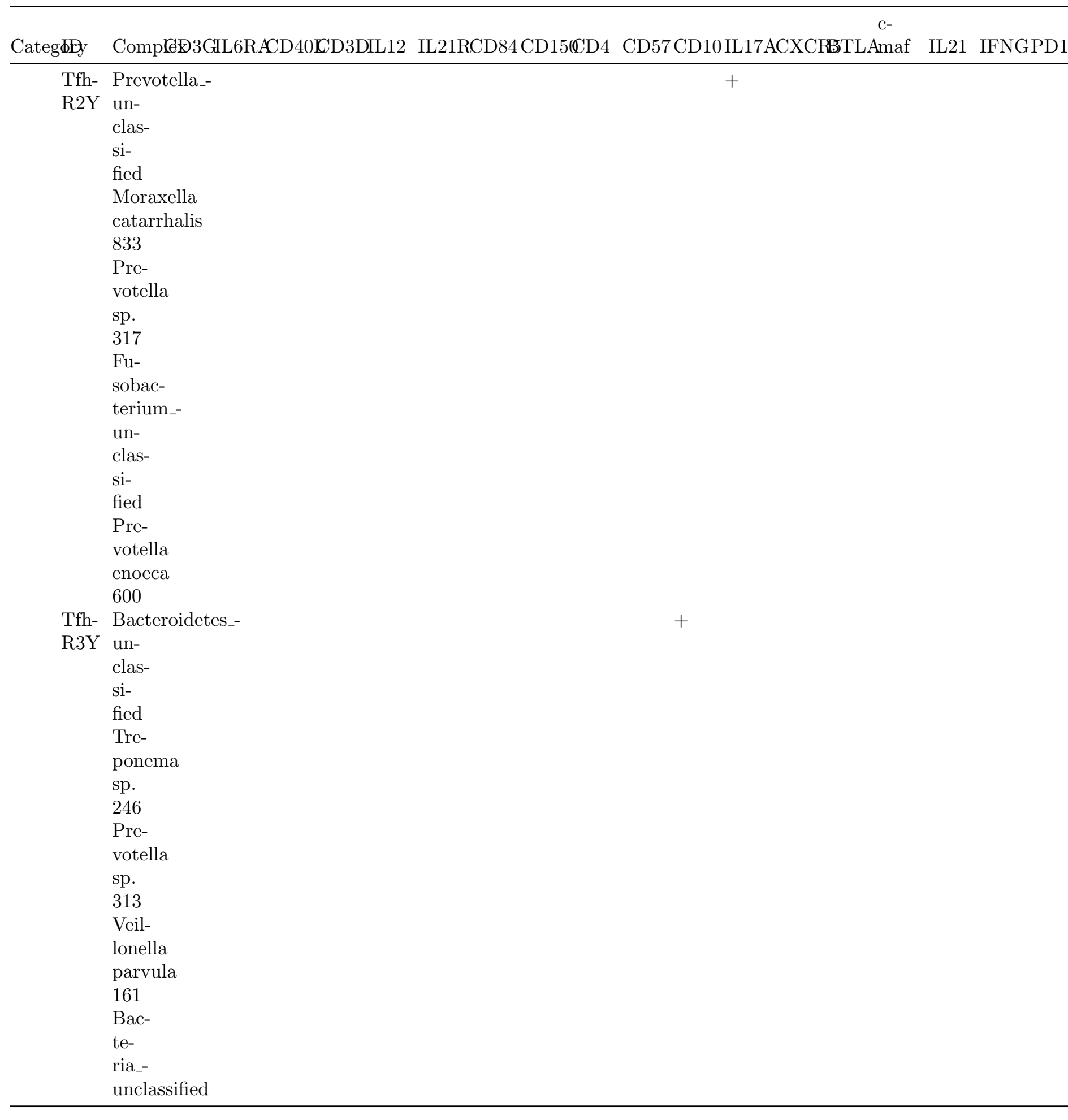

Table 3: Significant correlations of individual bacterial OTUs with Tfh genes in samples from both age groups and in health, disease, and resolution sites. + denotes a significant correlation between the OTU and gene expression levels, - no significant correlations, and NA signifies the OTU was not represented in the 
microbiome members that were evaluated.

\begin{tabular}{|c|c|c|c|c|c|c|}
\hline & ADULT & ADULT & ADULT & YOUNG & YOUNG & YOUNG \\
\hline Bacteria & Disease & Health & Resolution & Disease & Health & Resolution \\
\hline Fretibacterium sp. 361 & + & + & + & + & + & + \\
\hline Fretibacterium fastidiosum 363 & + & + & + & + & + & + \\
\hline Haemophilus sp. 035 & + & + & - & + & + & + \\
\hline Treponema unclassified & + & + & + & - & + & + \\
\hline Fretibacterium unclassified & + & - & + & + & + & + \\
\hline Prevotella sp. 526 & - & + & + & + & + & + \\
\hline Porphyromonas gingivalis 619 & + & + & + & + & - & + \\
\hline Chloroflexi_[G-1] sp. 439 & - & + & + & + & + & + \\
\hline Fusobacterium unclassified & + & - & + & + & + & - \\
\hline Leptotrichia_unclassified & + & + & + & - & - & + \\
\hline Treponema denticola 584 & + & - & + & - & + & + \\
\hline Porphyromonas sp. 279 & + & + & + & - & - & + \\
\hline Prevotella enoeca 600 & + & + & - & + & + & - \\
\hline Gemella morbillorum 046 & + & + & + & - & - & + \\
\hline Treponema sp. 246 & + & + & + & - & - & + \\
\hline Bacteria_unclassified & - & + & - & - & + & + \\
\hline Veillonella unclassified & + & + & + & - & - & - \\
\hline Prevotella unclassified & - & + & + & + & - & - \\
\hline A. actinomycetemcomitans 531 & - & + & + & - & + & - \\
\hline Streptococcus sp. 058 & + & - & + & - & - & + \\
\hline Treponema maltophilum 664 & + & + & - & - & + & - \\
\hline Porphyromonas endodontalis 273 & + & - & + & - & - & + \\
\hline Eubacterium infirmum 105 & - & + & + & + & - & - \\
\hline Prevotella sp. 820 & - & + & - & + & + & - \\
\hline Prevotella sp. 304 & - & - & + & + & - & + \\
\hline Prevotella intermedia 643 & - & + & + & - & + & - \\
\hline Leptotrichia sp. 223 & + & - & + & + & - & - \\
\hline Prevotella sp. 317 & + & - & - & + & - & + \\
\hline Filifactor alocis 539 & - & + & - & + & - & + \\
\hline Peptostreptococcaceae_[XIII]_unclassified & + & + & - & - & - & + \\
\hline Moraxella catarrhalis 833 & + & + & + & - & - & - \\
\hline Selenomonas_unclassified & - & + & + & - & - & - \\
\hline Streptococcus_unclassified & - & + & + & - & - & - \\
\hline Veillonella parvula 161 & - & - & + & - & + & - \\
\hline Selenomonas sputigena 151 & + & + & - & - & - & - \\
\hline Catonella morbi 165 & - & - & + & - & - & + \\
\hline Capnocytophaga_unclassified & - & - & + & - & - & + \\
\hline Veillonellaceae_[G-1] & - & - & - & + & - & + \\
\hline Fusobacterium sp._oral_taxon_203 & + & - & + & - & - & - \\
\hline Veillonellaceae_[G-1] sp. 155 & + & - & - & - & - & + \\
\hline Prevotella fusca 782 & - & - & + & - & - & + \\
\hline Pyramidobacter piscolens 357 & - & + & - & - & - & - \\
\hline Prevotella sp. 313 & - & - & - & - & + & - \\
\hline Streptococcus parasanguinis II 411 & - & - & + & - & - & - \\
\hline Aggregatibacter unclassified & - & + & - & NA & NA & NA \\
\hline Pasteurellaceae_unclassified & - & - & - & - & - & + \\
\hline Bacteroidetes_unclassified & - & - & - & - & - & + \\
\hline
\end{tabular}




\begin{tabular}{lllllll}
\hline & ADULT & ADULT & ADULT & YOUNG & YOUNG & YOUNG \\
\hline SR1_[G-1] sp. 345 & - & - & - & - & - & + \\
Prevotella sp. 311 & - & - & + & NA & NA & NA \\
Neisseria oralis 014 & - & + & - & - & - & - \\
Desulfobulbus sp. 041 & + & - & - & NA & NA & NA \\
Megasphaera micronuciformis 122 & - & - & - & NA & NA & NA \\
Porphyromonadaceae & - & - & - & - & - & - \\
Peptostreptococcaceae_[XI]_unclassified & - & - & - & NA & NA & NA \\
Veillonella dispar 160 & - & - & - & NA & NA & NA \\
Treponema socranskii 769 & - & - & - & NA & NA & NA \\
Prevotella sp. 443 & - & - & - & NA & NA & NA \\
Prevotella denticola 291 & - & - & - & NA & NA & NA \\
TOTAL & 25 & 29 & 32 & 17 & 17 & 28 \\
\hline
\end{tabular}

\section{Figure Legends}

Figure 1: Expression levels of Tfh genes in gingival tissues from young (A ) and adult (B ) animals. The data are expressed as fold-change in expression with disease $(0.5,1,3$ months) and resolution (5 months) compared to baseline healthy samples for each animal. The bars denote group means. The asterisk and horizontal bracket signifies significant difference from baseline at $\mathrm{p}<0.01$.

Figure 2: Expression of master transcription factor genes for various subsets of $\mathrm{CD}^{+} \mathrm{T}$ help cells [Tbet/TXB21 - Th1, GATA3 - Th2, ROR $\alpha / \gamma$ - Th17, FOXO1 - Th9, FOXP3 - Treg, BCL6 - Tfh cells]. Points denote group means for young and adult animals at each time point expressed as fold-change from baseline. Asterisk denotes significantly different from baseline at $\mathrm{p}<0.01$.

Figure 3: Z-score values for Tfh surface receptors (A \& B ), cytokines/chemokines (C \& D ), and transcription factors $(\mathbf{E})$ in adult and young animal gingival samples obtained in health (BL), during disease (0.5, 1,3 months) and clinical resolution (5 months). Points denote normalized group means comparing zscores for expression of a specific gene across all animals and timepoints.

Figure 4: Heatmap of correlations between bacterial OTUs relative abundance and individual gene expression young healthy (A), diseased (B ), and resolution (C) samples. Boxes provide estimate of gene and assigned bacterial complex interactions.

Figure 5: Heatmap of correlations between bacterial OTUs relative abundance and individual gene expression adult healthy (A ), diseased (B ), and resolution (C ) samples. Boxes provide estimate of gene and assigned bacterial complex interactions.

Figure 6: Schematic of potential protein interactome among Tfh associated genes. Blue nodes are surface receptors, green nodes are transcription factors, and orange nodes are secreted products. The symbols identify significant positive or negative correlations of the gene with bacterial complexes in healthy $(\mathrm{H})$, diseased (D), and resolution (R) samples from adult and young animal gingival tissues. Both denotes that individual bacteria within the complex demonstrated both significant positive and negative correlations the particular gene.

\section{Hosted file}

Tfh gene manu v8 7_20.pptx available at https://authorea.com/users/355423/articles/478655gingival-transcriptomics-of-follicular-t-cell-footprints-in-progressing-periodontitis 